\title{
Population Genetic Analysis of Phytophthora parasitica From Tobacco in Chongqing, Southwestern China
}

\author{
Qiang Zhang, ${ }_{1,2}$ Ruirui Feng, ${ }^{1,2}$ Qing Zheng, ${ }^{1,2}$ Jinyang Li, ${ }^{1,2}$ Zhirou Liu, ${ }^{1,2}$ Dan Zhao,, 3 \\ Yuling Meng, ${ }^{1,3}$ Yuee Tian, ${ }^{1,2}$ Weiwei Li, ${ }^{1,2}$ Xiaowei Ma, ${ }^{1,2}$ Shuang Wang, ${ }^{1,2}$ and Weixing Shan ${ }^{1,3, \dagger}$ \\ ${ }^{1}$ State Key Laboratory of Crop Stress Biology for Arid Areas, Northwest A\&F University, Yangling, Shaanxi 712100, China \\ ${ }^{2}$ College of Plant Protection, Northwest A\&F University, Yangling, Shaanxi 712100, China \\ ${ }^{3}$ College of Agronomy, Northwest A\&F University, Yangling, Shaanxi 712100, China
}

\begin{abstract}
Tobacco black shank, caused by Phytophthora parasitica, is one of the most notorious tobacco diseases and causes huge economic losses worldwide. Understanding the genetic variation of $P$. parasitica populations is essential to the development of disease control measures. In this research, 210 simple sequence repeat (SSR) markers for $P$. parasitica were identified, 10 of which were polymorphic among nine reference strains. We further performed population genetic analysis of $245 \mathrm{P}$. parasitica isolates randomly collected from tobacco fields in Chongqing for mating type, mo-

SSR genotyping distinguished $245 P$. parasitica isolates into 46 genotypes, four of which were dominant in the population. Low genotypic diversity and excess heterozygosity were common in nearly all of the populations from Chongqing. Population analysis showed that no differentiation existed among different populations. All isolates tested were highly sensitive to metalaxyl. Taken together, our results showed that the $P$. parasitica populations from tobacco fields in Chongqing belonged to a clonal lineage and were highly sensitive to metalaxyl.
\end{abstract} lecular variation at 14 SSR loci (four of which were identified previously), and sensitivity to the fungicide metalaxyl. The results showed that the A2 mating type was dominant and no A1 mating type isolate was discovered.
Keywords: simple sequence repeat, Phytophthora parasitica, pathogen diversity, metalaxyl sensitivity
Phytophthora parasitica Dastur (syn. P. nicotianae Breda de Haan), one of the most notorious oomycete plant pathogens, was first discovered as the pathogen triggering citrus root rot and gummosis and tobacco black shank (Kamoun et al. 2015). P. parasitica is a typical soilborne pathogen with a broad host spectrum comprising more than 255 plant genera in 90 families (Cline et al. 2008). Tobacco black shank caused by $P$. parasitica is one of main limiting factors for tobacco production worldwide, including in Chongqing, a mountainous region in Southwestern China.

Because oomycete pathogens are phylogenetically distant from fungi, they are insensitive to most fungicides. The acylalanine fungicide metalaxyl is a member of the few chemical agents used for control of diseases caused by Phytophthora spp. However, resistant strains were discovered soon after its use in several Phytophthora species. For example, metalaxyl-resistant $P$. parasitica isolates from burley tobacco were reported in Korea (Kang 2000). However, no resistant $P$. parasitica isolates have been found in Southwestern China, including in Guizhou (Wang et al. 2013) and Chongqing (Qian 2011).

$P$. parasitica can reproduce sexually and asexually, and the oospores resulting from sexual reproduction have been observed under laboratory conditions (Ko 1978). Coexistence of A1 and A2 mating type isolates allows the completion of the sexual cycle. Sexual

${ }^{\dagger}$ Corresponding author: W. Shan; wxshan@nwafu.edu.cn

Funding: This work was supported by the Chongqing Tobacco Major Program of Integrated Pest Management (no. NY20140401070002) and the Programme of Introducing Talents of Innovative Discipline to Universities (Project 111) from the State Administration of Foreign Experts Affairs (\#B18042) "Crop breeding for disease resistance and genetic improvement".

*The $\boldsymbol{e}$-Xtra logo stands for "electronic extra" and indicates that four supplementary tables are published online.

The author(s) declare no conflict of interest.

Accepted for publication 25 April 2019.

C 2019 The American Phytopathological Society reproduction leads to gene recombination in the progeny, which can incorporate favorable mutations and eliminate harmful ones (Crow 1994), and may result in better adaptability to the variable environment. However, the mating type distribution of $P$. parasitica populations from tobacco fields around the world was reported, which indicated that mostly only one mating type is found in the field (Gallup et al. 2006). Both A1 and A2 mating types were found in Virginia but A2 was dominant (96\%) (Parkunan et al. 2010). The A2 mating type was reported to be dominant in Anhui (Ma and Gao 2006) and Yunnan (Wang et al. 1997), China, whereas the A2 and A0 isolates (which do not produce oospores with either A1 or A2 mating type strains) coexisted in equal proportion and no A1 isolates were found in Chongqing (Sun 2009). However, Qian (2011) reported that A2 isolates were dominant in Chongqing, suggesting that the mating type distribution in Chongqing may have undergone a shift in the past 10 years.

Molecular genetic markers make it possible to track and trace individual genotypes and to improve understanding of pathogen population diversity (Parker et al. 1998). Simple sequence repeat (SSR) markers are one of the most useful molecular markers in population genetic analysis and have the following advantages: good reproducibility, codominance, low DNA template limitation, and low technical difficulty. SSR markers have been widely used in population analyses of Phytophthora pathogens, such as P. infestans (Flier et al. 2003; Grünwald et al. 2001; Li et al. 2012, 2013; Montarry et al. 2010; Montes et al. 2016; Tian et al. 2015a, b, 2016), P. cinnamomi (Pagliaccia et al. 2013), and P. ramorum (Eyre et al. 2013; Ivors et al. 2006). SSR markers have also been used for genotyping and population analysis of $P$. parasitica (Biasi et al. 2015, 2016; $\mathrm{Li}$ et al. 2017). Because of these advantages, SSR markers were used in this research to analyze the $P$. parasitica population from Chongqing.

Investigating the population structure and genetic diversity of $P$. parasitica populations facilitates a better understanding of the life cycle, primary infection sources, and evolution of the pathogen, which are important for developing effective disease management practices. $P$. parasitica populations in Chongqing have not yet been studied in depth. The aim of this research is to examine the genetic diversity of $P$. parasitica populations in tobacco fields in 
Chongqing, including mating type, metalaxyl sensitivity, and SSR maker-based determination of genetic diversity and mode of reproduction of the pathogen.

\section{Materials and Methods}

Sampling and isolation of $\boldsymbol{P}$. parasitica. Diseased tobacco samples were collected from six counties in Chongqing and were regarded as independent populations (Wulong, Qianjiang, and Youyang in 2014 and Wulong, Qianjiang, Fengjie, Wushan, and Pengshui in 2015, hereafter referred to as WL2014, QJ2014, YY2014, WL2015, QJ2015, FJ2015, WS2015, and PS2015, respectively). Pathogen isolation and purification were performed in the laboratory. The typical disc-like pith tissues were placed on a $5 \% \mathrm{CA}$ agar plate $(50 \mathrm{ml}$ of carrot extract, $0.1 \mathrm{~g}$ of $\mathrm{CaCO}_{3}, 0.01 \mathrm{~g}$ of $\beta$-sitosterol, and $8 \mathrm{~g}$ of agar/liter) with the proper antibiotics $(100 \mu \mathrm{g} / \mathrm{ml}$ of ampicillin, $10 \mu \mathrm{g} / \mathrm{ml}$ of rifampicin, and $9 \mu \mathrm{g} / \mathrm{ml}$ of nystatin) directly. For those without the typical disclike pith tissues, a small piece of pith tissue of new developing lesions was surface disinfected in $75 \%$ ethanol for $30 \mathrm{~s}$, rinsed in sterilized water three times, and placed on 5\% CA agar plates with the proper antibiotics $(100 \mu \mathrm{g} / \mathrm{ml}$ of ampicillin, $10 \mu \mathrm{g} / \mathrm{ml}$ of rifampicin, and $9 \mu \mathrm{g} / \mathrm{ml}$ of nystatin). All isolates were incubated at $23^{\circ} \mathrm{C}$ in the dark and identified as $P$. parasitica based on morphological characters. Mature cultures of isolates were covered with sterile liquid paraffin and stored at $16^{\circ} \mathrm{C}$ as previously described (Onions 1971).

Mating type determination. Mating types were determined by pairing unknown isolates with known A1 and A2 mating type strains of P. parasitica $\mathrm{Pp} 007$ and Pp018, respectively, on 10\% V8 juice agar plates with $0.02 \mathrm{~g} /$ liter of $\beta$-sitosterol. After incubation at $23^{\circ} \mathrm{C}$ in the dark for about 10 days, oospores were surveyed under a microscope. Isolates were also paired with themselves to examine whether they were self-fertile.

DNA extraction. $P$. parasitica mycelium was harvested after incubation in $5 \% \mathrm{CA}$ broth in the dark at $23^{\circ} \mathrm{C}$ for 4 days. Liquid nitrogen freeze-dried mycelium was ground to a fine powder. Genomic DNA of $P$. parasitica was extracted following the protocol as previously described (Goodwin et al. 1992; Tian et al. 2015a) with minor modifications. Briefly, the mycelium powder was dissolved thoroughly in the DNA extraction buffer (7 M of urea, $350 \mathrm{mM}$ of $\mathrm{NaCl}$, $4 \mathrm{mM}$ of EDTA, $50 \mathrm{mM}$ of Tris- $\mathrm{HCl}, \mathrm{pH} 8.0,1 \%$ Na-lauryl sarcosine, and 5\% Tris-saturated phenol, $\mathrm{pH} 8.0$ ) and extracted with Trissaturated phenol, $\mathrm{pH} 8.0$, and chloroform. The DNA was precipitated with a half volume of $7.5 \mathrm{M}$ of $\mathrm{NH}_{4} \mathrm{OAC}$ and 1.5 volumes of isopropanol and was then washed in $75 \%$ ethanol twice. The air-dried DNA pellet was resuspended in $\mathrm{ddH}_{2} \mathrm{O}$ with $0.1 \mathrm{mg} / \mathrm{ml}$ of RNase $\mathrm{A}$ and the DNA solution was incubated at $37^{\circ} \mathrm{C}$ for $30 \mathrm{~min}$. Then the DNA was again extracted with phenol and chloroform and precipitated with $\mathrm{NH}_{4} \mathrm{OAC}$ and isopropanol as above. Finally, the air-dried DNA pellet was dissolved in $30 \mu \mathrm{L}$ of $\mathrm{ddH}_{2} \mathrm{O}$ and stored at $-80^{\circ} \mathrm{C}$ for later use.

Development of SSR markers. To identify SSR markers suitable for $P$. parasitica population genetic analyses, the publicly available $P$. parasitica reference genome INRA-310 version 2.0 (GenBank assembly accession number GCA_000247585.2) was used to scan for candidate SSR loci (2 to 6 bp per motif) with SSR Locator version 1 (da Maia et al. 2008). The parameters were set as 2 mers $(\times 10), 3$ mers $(\times 7), 4$ mers $(\times 5), 5$ mers $(\times 4)$, and 6 mers $(\times 4)$, respectively. Only perfect repeats were allowed during this search. More than 300 SSR loci were identified and 210 SSRs with the longest length were chosen. The primers flanking the selected SSR loci were designed with Primer3 (Untergasser et al. 2012). All of the primers designed were examined for polymorphism among nine reference $P$. parasitica strains (Supplementary Table S1) by PCR amplification followed by polyacrylamide gel electrophoresis (PAGE). In order to obtain enough SSR markers polymorphic in Chongqing populations, the candidates (polymorphic among the reference strains) from the last 145 primers (SSR66 to SSR210) were also examined for polymorphism among a subset of Chongqing isolates (90 randomly selected isolates). Those primers that showed high polymorphisms among reference strains (for the first 65 primers) or showed polymorphisms in Chongqing isolates (for the last 145 primers) were chosen for further analysis. To confirm the size of PCR products of different
$P$. parasitica strains, selected PCR products amplified with highfidelity DNA polymerase Pfu (Promega) were linked into T-vector pMD19 (simple) (Takara) and sequenced. Polymorphism information content (PIC) was calculated as:

$$
\mathrm{PIC}=1-\sum_{\mathrm{i}}{ }^{\mathrm{k}} P_{\mathrm{i}}{ }^{2}
$$

(Anderson et al. 1993; Botstein et al. 1980), where $P_{\mathrm{i}}$ is the frequency of the $i$ th allele in the examined $P$. parasitica strains and $\mathrm{k}$ is the total number of alleles for each SSR locus.

Population analysis of $\boldsymbol{P}$. parasitica using SSR markers. The obtained $P$. parasitica isolates were analyzed with 14 SSR markers, among which 10 were developed in this study and four were developed in previous reports (Biasi et al. 2015; Li et al. 2017). The size of the PCR products of different representative genotypes was confirmed as described above.

Considering that the large asexual reproductive ability and repetitive sample collection may influence the data analysis (Chen and McDonald 1996), we constructed a "clone-corrected data set" that included a representative isolate of each genotype in each population. This data set was used for the analysis of Weir and Cockerham's coefficient of differentiation $\theta$ among different populations, analysis of molecular variance (AMOVA), principal coordinate analysis (PCoA), and Nei's original measures of genetic distance. The full data set was used for calculating the genotype accumulation curve, genotypic diversity, and gene diversity analysis.

Genetic diversity was estimated by both genotypic diversity and gene diversity. Genotypic diversity is described by three indices: richness, diversity, and evenness. Given the difference among the sample number of each population, the genotypic richness was calculated based on the rarefaction curve (Grünwald et al. 2003). Genotypic diversity was estimated by $1-\lambda$ and $\lambda$ (Simpson's index lambda; Simpson 1949) is calculated as:

$$
\lambda=\sum\left[n_{\mathrm{i}} \times\left(n_{\mathrm{i}}-1\right)\right] /[N \times(N-1)]
$$

where $n_{\mathrm{i}}$ is the number of individuals of the $i$ th genotypes and $N$ is the sample size for each population. To reduce the influence of sample size, $1-\lambda$ was corrected as:

$$
1-\lambda=1-N /(N-1) \times \lambda
$$

Genotypic evenness was quantified by $E_{5}$ (Grünwald et al. 2003), which is less dependent on the number of genotypes in a sample. All of the indices above were calculated with the R package Poppr (Kamvar et al. 2014). Gene diversity was estimated by analyzing the observed heterozygosity $\left(H_{\mathrm{O}}\right)$, unbiased expected heterozygosity $\left(H_{\mathrm{E}}\right)$, and fixation index $\left(F_{\mathrm{IS}}\right) . H_{\mathrm{E}}$ and $H_{\mathrm{O}}$ values were calculated by POPGENE version 1.31 (Yeh et al. 1997) and fixation index values were computed as:

$$
F_{\mathrm{IS}}=1-H_{\mathrm{E}} / H_{\mathrm{O}}
$$

AMOVA was performed using GenAlEx 6.5 (Peakall and Smouse 2006, 2012). Weir and Cockerham's coefficient of differentiation $\theta$ was calculated with MULTILOCUS version 1.3 (Agapow and Burt 2001). These two analyses were used to test the null hypothesis that no difference exists between populations. PCoA was performed using GenAlEx 6.5 (Peakall and Smouse 2006, 2012) to unravel whether there was any population structure among multilocus genotypes (MLGs). Nei's original measures of genetic distance were performed with POPGENE version 1.31 (Yeh et al. 1997). The genotype accumulation curve was generated with the R package Poppr to test whether the SSR markers had enough discrimination power to differentiate genotypes.

To reveal the genetic relationship among the SSR genotypes detected in Chongqing and the nine reference strains, an unrooted neighbor-joining tree using Bruvo's distance (Bruvo et al. 2004) was constructed with the $\mathrm{R}$ package Poppr and visualized in Mega 6.0 (Tamura et al. 2013).

Determination of sensitivity of $P$. parasitica isolates to metalaxyl. Metalaxyl sensitivity was estimated with half maximal effective 
concentrations ( $\mathrm{EC}_{50}$ values), which means the dose for $50 \%$ mycelial growth inhibition. A stock metalaxyl solution was made by dissolving $0.104 \mathrm{mg}$ of $96 \%$ metalaxyl in $1 \mathrm{ml}$ of acetone. The stock was diluted with $\sim 40^{\circ} \mathrm{C}$ soluble $5 \% \mathrm{CA}$ agar medium to prepare plates of different final metalaxyl concentrations: $0,0.01,0.10,0.50,1.00,2.50,5.00$, and $10.00 \mu \mathrm{g} / \mathrm{ml}$. Selected $P$. parasitica isolates were grown on a $5 \%$ CA agar medium plate for 3 days in the dark and 6-mm diameter mycelial plugs were made around the edge of colony. One mycelial plug was placed in the center of the 5\% CA agar plate with different concentrations of metalaxyl and incubated at $23^{\circ} \mathrm{C}$ in the dark for 3 days. The colony diameter was measured and $\mathrm{EC}_{50}$ values were calculated. Based on the $\mathrm{EC}_{50}$ value, the isolates were regarded as resistant $\left(\mathrm{EC}_{50} \geq 100 \mu \mathrm{g} / \mathrm{ml}\right)$, intermediate $\left(100>\mathrm{EC}_{50} \geq 1 \mu \mathrm{g} / \mathrm{ml}\right)$, or sensitive $\left(\mathrm{EC}_{50}<1 \mu \mathrm{g} / \mathrm{ml}\right)$ (Wang et al. 2013).

\section{Results}

Identification of SSR markers. Based on the publicly available P. parasitica genome, 210 candidate loci were identified and selected for further analysis (Supplementary Table S2). Examination for polymorphism among nine $P$. parasitica strains isolated from different hosts or origins showed that 10 of 210 SSR markers were polymorphic (Table 1). All of the markers selected were distributed in different supercontigs (Table 1), suggesting their independent inheritance. Sequencing of the PCR products from representative genotypes

Table 1. Simple sequence repeat (SSR) markers developed for Phytophthora parasitica

\begin{tabular}{|c|c|c|c|c|}
\hline SSR marker & SSR primer $^{\mathrm{a}}$ & Scaffold $^{\mathrm{b}}$ & Target size (bp) & Motif \\
\hline $\mathrm{SSR} 01^{\mathrm{c}}$ & $\begin{array}{l}\text { F: CGGTATGGAGCCGAAATAGA } \\
\text { R: GCCCTAAGTGGCTGATGAAG }\end{array}$ & Supercontig_2.9 & 187 & ACTC \\
\hline SSR08 ${ }^{c}$ & $\begin{array}{l}\text { F: AACAATGCTGCTGTGAGTGC } \\
\text { R: CTCACACCATTGGACACTGC }\end{array}$ & Supercontig_2.3 & 230 & $(\mathrm{AGGCAC}) \mathrm{x}-(\mathrm{CAAGCT}) \mathrm{y}$ \\
\hline $\operatorname{SSR} 25^{\mathrm{c}}$ & $\begin{array}{l}\text { F: TTTGTCCTCAGATCCCAAGG } \\
\text { R: GCCCACGTCCTCTAGATTCA }\end{array}$ & Supercontig_2.23 & 188 & CTGA \\
\hline $\operatorname{SSR} 30^{c}$ & $\begin{array}{l}\text { F: ACGTCGGCTTCAACTCTGAT } \\
\text { R: AACAGCCTATCAGATCGCGT }\end{array}$ & Supercontig_2.6 & 250 & TCTG \\
\hline $\operatorname{SSR} 42^{c}$ & $\begin{array}{l}\text { F: GCTCAACAAGTCATACGGCA } \\
\text { R: CGCGAGACCGTAGCTTTATC }\end{array}$ & Supercontig_2.113 & 213 & CAG \\
\hline $\operatorname{SSR} 48^{\mathrm{c}}$ & $\begin{array}{l}\text { F: GTGCAGTTGAGGCACAAAGA } \\
\text { R: CGTGACCGTTAATTTGGCTT }\end{array}$ & Supercontig_2.5 & 247 & TTAG \\
\hline SSR $122^{c}$ & $\begin{array}{l}\text { F: CATGGTTAAGAAAATGAGAACAATG } \\
\text { R: TGATGTTTTTGGCTGGTTGA }\end{array}$ & Supercontig_2.25 & 123 & AAGA \\
\hline SSR $141^{\mathrm{c}}$ & $\begin{array}{l}\text { F: CTCAGTCAGTCAGCCACTCG } \\
\text { R: TGTGTTGCACGCAAGTTTTT }\end{array}$ & Supercontig_2.49 & 250 & CTCA \\
\hline SSR166 & $\begin{array}{l}\text { F: TAACGACTCGATCCCAGGTC } \\
\text { R: TTTGTGGAAATTGAAATGCG }\end{array}$ & Supercontig_2.71 & 232 & $\mathrm{CA}$ \\
\hline SSR $182^{c}$ & $\begin{array}{l}\text { F: TTGGTGTTCTCGTGTCTTGG } \\
\text { R: TTGCGGTCAGAATTGACAAG }\end{array}$ & Supercontig_2.215 & 233 & GTA \\
\hline $\operatorname{Pn} 251^{\prime d}$ & $\begin{array}{l}\text { F: CAGCTGAAGCGTAACGACAG } \\
\text { R: ATAAGTGAGGGAGGGAGGGA }\end{array}$ & Supercontig_2.196 & 219 & CTCA \\
\hline$P 5^{\prime d}$ & $\begin{array}{l}\text { F: AACTTCGAGTCTCGTCACGG } \\
\text { R: CTCCGAGGTCCAAATGTGAT }\end{array}$ & Supercontig_2.21 & 227 & TCTG \\
\hline $\mathrm{P} 15^{\mathrm{f}}$ & $\begin{array}{l}\text { F: AGCTTCTGCAGTAACGGTAA } \\
\text { R: CGATCAAAGATTACTGCAACT }\end{array}$ & Supercontig_2.7 & 110 & GTA \\
\hline $\mathrm{P} 17^{\mathrm{f}}$ & $\begin{array}{l}\text { F: GTCCTCAGGGATCAGCACAT } \\
\text { R: TGGATATCGTTCCCGTTGTT }\end{array}$ & Supercontig_2.53 & 147 & GTT \\
\hline
\end{tabular}

${ }^{\mathrm{a}} \mathrm{F}=$ forward and $\mathrm{R}=$ reverse.

b The scaffold is based on the $P$. parasitica INRA-310 genome sequence (version 2.0).

${ }^{c}$ Primers developed in this research.

${ }^{\mathrm{d}} \mathrm{Pn} 251^{\prime}$ and P5' were redesigned based on SSR loci Pn251 and P5 published previously (Li et al. 2017).

e Same as P5 reverse primer in Biasi et al. (2015).

${ }^{\mathrm{f}}$ Primers reported in Biasi et al. (2015).

Table 2. Polymorphism analysis of new simple sequence repeat (SSR) markers among nine Phytophthora parasitica strains

\begin{tabular}{|c|c|c|c|c|c|c|c|c|c|c|}
\hline SSR locus & Pp002 & Pp007 & Pp009 & Pp014 & Pp016 & Pp018 & Pp019 & Pp023 & Pp025 & PIC $^{\mathbf{a}}$ \\
\hline SSR01 & $167 / 191^{\mathrm{b}}$ & $175 / 183$ & $179 / 195$ & $179 / 179$ & $179 / 179$ & $183 / 187$ & $183 / 183$ & $155 / 195$ & $159 / 183$ & 0.81 \\
\hline SSR08 & $224 / 248$ & $230 / 230$ & $230 / 230$ & $230 / 230$ & $230 / 230$ & $230 / 230$ & $\mathrm{ND}^{\mathrm{c}}$ & ND & $218 / 230$ & 0.47 \\
\hline SSR 25 & $170 / 190$ & $170 / 190$ & $170 / 170$ & $170 / 190$ & $170 / 190$ & $170 / 190$ & $170 / 190$ & $170 / 170$ & $170 / 170$ & 0.44 \\
\hline SSR30 & $246 / 246$ & $238 / 250$ & $242 / 242$ & $238 / 250$ & $238 / 250$ & $242 / 250$ & $242 / 250$ & $242 / 242$ & $242 / 242$ & 0.69 \\
\hline SSR42 & $210 / 210$ & $201 / 213$ & $210 / 210$ & $201 / 213$ & $201 / 213$ & $201 / 210$ & $201 / 210$ & $210 / 210$ & $210 / 210$ & 0.59 \\
\hline SSR48 & $181 / 185$ & $185 / 189$ & $177 / 189$ & $185 / 189$ & $185 / 189$ & $181 / 185 / 189^{d}$ & $181 / 185 / 189$ & $181 / 197$ & $181 / 193 / 217$ & 0.77 \\
\hline SSR122 & $131 / 131$ & $119 / 123$ & $127 / 127$ & $119 / 123$ & $119 / 123$ & $119 / 119$ & $119 / 119$ & $127 / 131$ & $147 / 147$ & 0.75 \\
\hline SSR141 & $250 / 250$ & $238 / 250$ & $246 / 250$ & $238 / 250$ & $238 / 250$ & $250 / 250$ & ND & $250 / 250$ & $250 / 250$ & 0.40 \\
\hline SSR166 & $224 / 224$ & $220 / 220$ & $220 / 224$ & $220 / 232$ & $220 / 232$ & $220 / 224$ & $220 / 224$ & $220 / 224$ & $220 / 224$ & 0.59 \\
\hline SSR182 & $216 / 216$ & $216 / 216$ & $204 / 204$ & $216 / 216$ & $216 / 216$ & $216 / 216$ & $216 / 216$ & $216 / 216$ & $216 / 216$ & 0.20 \\
\hline
\end{tabular}

${ }^{\mathrm{a}} \mathrm{PIC}=1-\sum_{\mathrm{i}}^{\mathrm{k}} P_{\mathrm{i}}^{2}$, where $P_{\mathrm{i}}$ is the frequency of $i$ th allele in nine reference strains.

$\mathrm{b}$ The allele length (in basepairs) for each locus in different isolates.

${ }^{\mathrm{c}} \mathrm{ND}=$ not determined.

${ }^{d}$ The presence of three alleles suggests that the strain may be of segmental polyploidy, which was also reported in Biasi et al. (2015). 
revealed that the variation resulted from copy number differences of SSR motifs (Table 2). Results from PIC value calculation revealed that all of the selected SSR markers showed high polymorphism with PIC (>0.40, except SSR182) (Table 2).

Four reported SSR loci (Pn251, P17, P15, and P5) (Biasi et al. 2015; Li et al. 2017) were also used to analyze the P. parasitica populations from Chongqing, which were distributed in four supercontigs different from the SSR loci identified in this research (Table 1).

Isolation and mating type determination of $P$. parasitica. A total of $245 P$. parasitica isolates were obtained from diseased tobacco plants in two consecutive years (Fig. 1). In 2014, 96 diseased tobacco plants were collected from three counties and 78 isolates were obtained, comprising 13, 38, and 27 isolates for Wulong, Qianjiang and Youyang, respectively. In 2015, 167 isolates were obtained from 186 diseased tobacco plants from five counties, comprising 26, 42, 24, 33, and 42 isolates for Wulong, Pengshui, Qianjiang, Fengjie, and Wushan, respectively (Fig. 1).

All $P$. parasitica isolates were examined for mating type. The results showed that no A1 mating type or self-fertile isolates were detected and nearly all isolates $(99.6 \%)$ were of the A2 mating type, except one isolated in 2015 was of the A0 mating type (Fig. 1).

SSR genotyping of $P$. parasitica populations. A total of 46 multilocus SSR genotypes were detected from $245 P$. parasitica isolates sampled in two consecutive years (78 and 167 isolates for 2014 and 2015, respectively) based on 14 SSR markers (nine of which were polymorphic in the $P$. parasitica population from Chongqing) (Supplementary Table S3). The genotype accumulation curve showed that these nine polymorphic SSR markers had enough discrimination power to differentiate genotypes (Fig. 2). Eight genotypes were unique in 2014 and 34 were unique in 2015. There were four shared identical SSR genotypes (MLG1, MLG2, MLG5, and MLG7) in both years (Fig. 3).

Four SSR genotypes (MLG1, MLG2, MLG3, and MLG4) were dominant in P. parasitica populations, with a frequency of 20.82 , $17.14,11.43$, and $10.61 \%$, respectively. Forty genotypes occurred at a very low frequency $(<5 \%), 27$ of which were singletons. Apart from WL2015 and PS2015, all of the other populations were dominated by single genotypes, with MLG1 being dominant in WL2014 and YY2014, MLG2 being dominant in Qianjiang in both years, MLG3 being dominant in WS2015, and MLG4 being dominant in FJ2015 (Fig. 3).

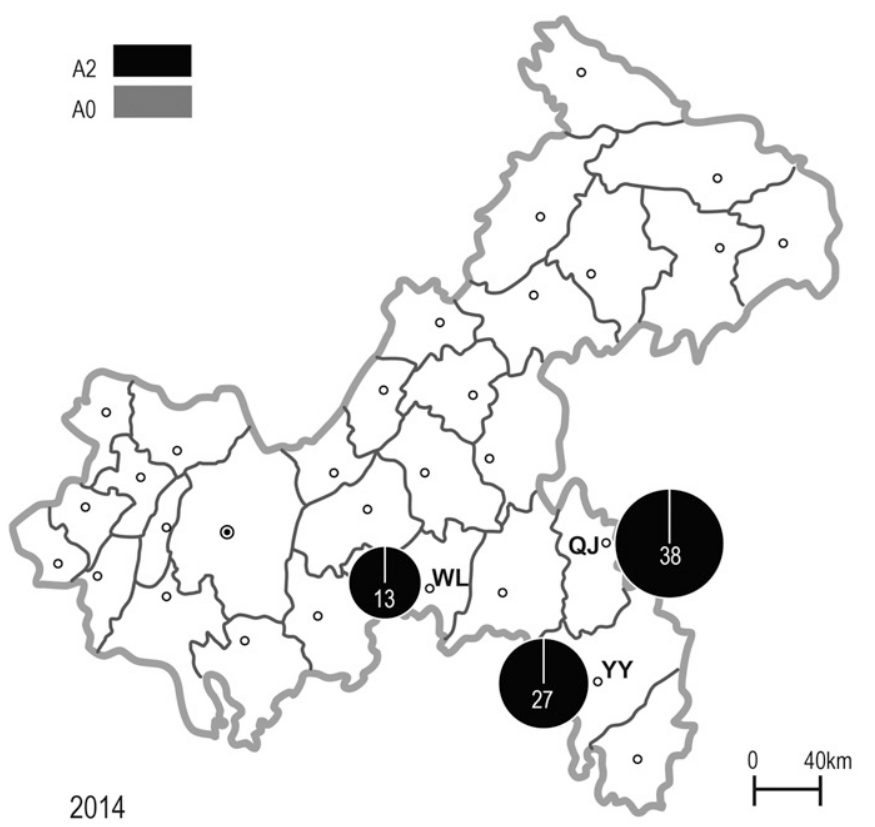

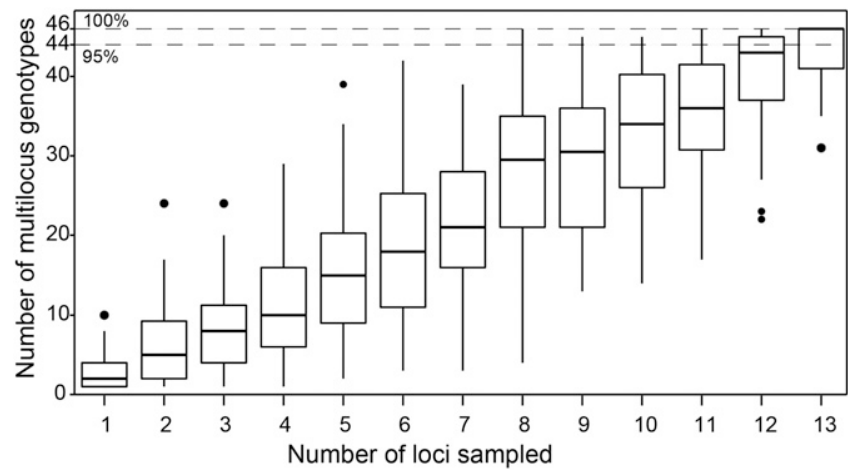

Fig. 2. Genotype accumulation curve for the Phytophthora parasitica population from Chongqing.

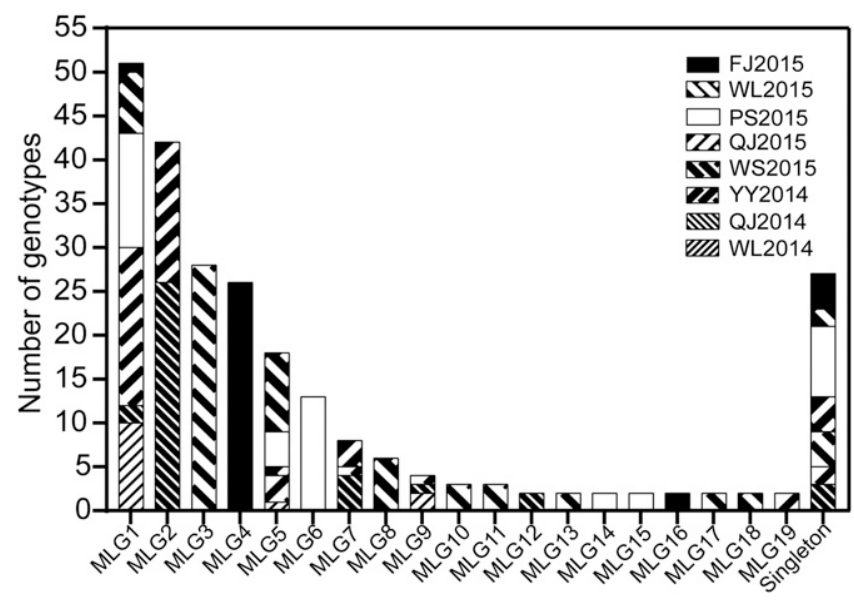

Fig. 3. Genotype distribution of Phytophthora parasitica populations in Chongqing. MLG1 to MLG19 indicate the 19 simple sequence repeat (SSR) multilocus genotypes detected more than once. 2014 and 2015 indicate the years of sample collection from the following: FJ $=$ Fengjie, $W L=$ Wulong, $P S=$ Pengshui, $Q J=$ Qianjiang, WS = Wushan, and $Y Y=$ Youyang.

Fig. 1. Sampling locations and mating type distribution of Phytophthora parasitica populations from Chongqing. The total number of $P$. parasitica isolates for each region is marked in the center and the circle size reflects relative sample sizes from different regions. The obtained $P$. parasitica isolates were paired with known mating type strains of $\mathrm{A} 1$ (Pp007) and A2 (Pp018) on 10\% V8 juice agar plates supplemented with 0.02 g/liter $\beta$-sitosterol. WL $=$ Wulong, YY $=$ Youyang, QJ = Qianjiang, FJ = Fengjie, WS $=$ Wushan, and PS $=$ Pengshui. 
Genotypic and gene diversities. The genotypic richness among populations was similar, ranging from 3.00 to 6.32 . The genotypic diversity index $1-\lambda$ showed a low level of genotypic diversity in all of the populations (varying from 0.383 to 0.554 ) except that of WL2015 and PS2015. Genotypic evenness estimated with $E_{5}$ was not highly variable among all of the populations (ranging from 0.413 to 0.624 ) except that of WL2015. Compared with other populations, the PS2015 population showed higher evenness (i.e., 0.810) and the WL2015 population showed both higher diversity (i.e., 0.775) and evenness (i.e., 0.834). In all populations, the observed heterozygosity was higher than the expected heterozygosity, resulting in a negative fixation index $F_{\text {IS }}$. The $F_{\text {IS }}$ values were not highly variable in all populations, varying from -0.920 to -0.833 (Table 3).

Genetic structure analysis. The AMOVA showed that the major variance was within populations $(98 \%$, estimated variance $=4.950)$, whereas the remaining variance $\left(2 \%\right.$, estimated variance $=0.100, F_{\text {st }}$ value $=0.035, P=0.099)$ was among populations. The estimation of Weir and Cockerham's coefficient of differentiation $\theta$ (ranging from -0.130 to 0.022 ) accepted the null hypothesis that there was no significant differentiation among populations in the same year, nor between populations from the same region in different years (Table 4). The Nei's genetic distance between populations ranged from 0.003 to 0.063 (Table 4). PCoA showed that there was no association between geographic regions and MLGs.

$P$. parasitica isolates from Chongqing were genetically different from the reference strains. To reveal the genetic relationship between $P$. parasitica isolates from Chongqing and those from other regions, a neighbor-joining tree was constructed based on 10 shared SSR markers (SSR01, SSR08, SSR25, SSR30, SSR42, SSR48, SSR122, SSR141, SSR166, and SSR182) using Bruvo's distance. The results showed that all 46 MLGs from Chongqing were genetically similar to each other and formed a unique cluster. The isolates from Zhejiang Province, China, and Australia were genetically distant from that of Chongqing and formed the other cluster (Fig. 4).

$P$. parasitica isolates from Chongqing were sensitive to metalaxyl. To examine sensitivity of $P$. parasitica isolates to fungicides, we randomly selected an isolate representing each multilocus SSR genotype (excluding MLG34 and MLG35, which did not grow well on the CA agar plates) and tested their tolerance to the commonly used fungicide metalaxyl. Results revealed by $\mathrm{EC}_{50}$ values showed that all tested isolates were sensitive to metalaxyl $\left(\mathrm{EC}_{50}<1 \mu \mathrm{g} / \mathrm{ml}\right)$, with $\mathrm{EC}_{50}$ values ranging from 0.0520 to $0.3320 \mu \mathrm{g} / \mathrm{ml}$ (Supplementary Table S4).

\section{Discussion}

$P$. parasitica is a typical soilborne plant pathogen with a wide host range. The higher optimum growth temperature suggests that $P$. parasitica will gain more importance in the foreseeable future because of global warming (Kamoun et al. 2015). A better understanding of $P$. parasitica evolution and population dynamics will facilitate development of effective disease management strategies. In this study, we identified a new set of SSR markers and analyzed population structures of $P$. parasitica populations from tobacco fields from Chongqing.

SSR markers are widely used in plant pathogen population genetic analysis. Based on the publicly available $P$. parasitica genome, several SSR markers were identified and used for $P$. parasitica population diversity analysis (Biasi et al. 2015, 2016; Li et al. 2017). This research was started before the first study was published in 2015. A low level of polymorphisms was identified using these SSR markers among $90 P$. parasitica isolates from Chongqing tobacco fields, suggesting that the number of polymorphic SSR markers may not be sufficient for the genetic analysis. Therefore, we used both the markers published previously and newly identified in this research for the genetic analysis of $P$. parasitica populations from Chongqing. In this study, we performed a genome survey for development of SSR markers for $P$. parasitica population genetic research. We identified 210 SSR markers: 10 showed polymorphisms among nine $P$. parasitica strains isolated from different regions and different hosts. All of the SSR loci identified were distributed in different supercontigs, suggesting that they are not physically linked. Besides these 10 SSR markers used in this research, we also found 15 SSR markers that showed polymorphisms among nine reference strains with one or two clear bands during PAGE, which may also be candidate choices for other researchers to test in their

Table 3. Genotypic diversity and gene diversity analysis of Phytophthora parasitica populations from Chongqing in 2014 and 2015

\begin{tabular}{|c|c|c|c|c|c|c|c|c|}
\hline Index $^{a}$ & WL2014 $^{b}$ & QJ2014 & YY2014 & WS2015 & QJ2015 & PS2015 & WL2015 & FJ2015 \\
\hline$N$ & 13 & 38 & 27 & 42 & 24 & 42 & 26 & 33 \\
\hline$N G_{\mathrm{O}}$ & 3 & 8 & 7 & 9 & 7 & 13 & 6 & 7 \\
\hline$N G_{\mathrm{E}}$ & 3.00 & 4.34 & 4.54 & 4.66 & 4.63 & 6.32 & 4.75 & 3.61 \\
\hline $1-\lambda$ & 0.410 & 0.526 & 0.553 & 0.552 & 0.554 & 0.810 & 0.775 & 0.383 \\
\hline$E_{5}$ & 0.617 & 0.460 & 0.494 & 0.441 & 0.494 & 0.624 & 0.834 & 0.413 \\
\hline$H_{\mathrm{O}}$ & 0.714 & 0.712 & 0.712 & 0.711 & 0.714 & 0.711 & 0.714 & 0.714 \\
\hline$H_{\mathrm{E}}$ & 0.379 & 0.373 & 0.375 & 0.375 & 0.383 & 0.386 & 0.390 & 0.372 \\
\hline$F_{\mathrm{IS}}{ }^{\mathrm{c}}$ & -0.887 & -0.908 & -0.896 & -0.895 & -0.866 & -0.844 & -0.833 & -0.920 \\
\hline
\end{tabular}

${ }^{a} N=$ sample size, $N G_{\mathrm{O}}=$ number of observed multilocus genotypes, $N G_{\mathrm{E}}=$ number of expected multilocus genotype; $E_{5}=$ evenness of genotypes, $H_{\mathrm{O}}=$ the observed heterozygosity, $H_{\mathrm{E}}=$ Nei's expected heterozygosity (Nei 1973), and $F_{\mathrm{IS}}=$ fixation index.

b 2014 and 2015 indicate the years of sample collection from the following: WL = Wulong, QJ = Qianjiang, YY = Youyang, WS = Wushan, PS = Pengshui, and FJ $=$ Fengjie

${ }^{\mathrm{c}} F_{\mathrm{IS}}$ was calculated as follows: $F_{\mathrm{IS}}=1-H_{\mathrm{O}} / H_{\mathrm{E}} .1-\lambda$ was corrected for sample size and calculated as $1-\lambda=1-N /(N-1) \times \lambda$.

Table 4. Estimation of Weir and Cockerham's coefficient of differentiation $\theta$ and Nei's genetic distance among Phytophthora parasitica populations from Chongqing ${ }^{\mathrm{a}}$

\begin{tabular}{|c|c|c|c|c|c|c|c|c|}
\hline Populations & WL2014b & QJ2014 & YY2014 & WS2015 & QJ2015 & PS2015 & WL2015 & FJ2015 \\
\hline WL2014 & - & 0.018 & 0.003 & - & - & - & 0.023 & - \\
\hline QJ2014 & $-0.096^{\mathrm{ns}}$ & - & 0.014 & - & 0.007 & - & - & - \\
\hline YY2014 & $-0.130^{\mathrm{ns}}$ & $-0.047^{\mathrm{ns}}$ & - & - & - & - & - & - \\
\hline WS2015 & - & - & - & - & 0.062 & 0.048 & 0.063 & 0.039 \\
\hline QJ2015 & - & $-0.061^{\mathrm{ns}}$ & - & $0.022^{\mathrm{ns}}$ & - & 0.038 & 0.019 & 0.038 \\
\hline PS2015 & - & - & - & $0.022^{\mathrm{ns}}$ & $-0.001^{\mathrm{ns}}$ & - & 0.037 & 0.022 \\
\hline WL2015 & $-0.102^{\mathrm{ns}}$ & - & - & $0.017^{\mathrm{ns}}$ & $-0.054^{\mathrm{ns}}$ & $-0.009^{\mathrm{ns}}$ & - & 0.043 \\
\hline FJ2015 & - & - & - & $-0.008^{\mathrm{ns}}$ & $-0.020^{\mathrm{ns}}$ & $-0.024^{\mathrm{ns}}$ & $-0.019^{\mathrm{ns}}$ & - \\
\hline
\end{tabular}

a Above the diagonal is Nei's genetic distance (Nei 1972), and below the diagonal is the $\theta$ value. Dashes indicate that the indices were not calculated.

b 2014 and 2015 indicate the years of sample collection from the following: WL = Wulong, QJ = Qianjiang, YY = Youyang, WS = Wushan, PS = Pengshui, and $\mathrm{FJ}=$ Fengjie. $\mathrm{ns}=P>0.05$ indicates no significant difference. 
$P$. parasitica populations. In this research, the polymorphisms of candidate SSR loci were analyzed among nine $P$. parasitica reference strains. These strains are diverse in terms of host origin and geographic location, which is suggestive of a certain level of diversity required for the detection of polymorphism and the development of genetic markers. During analysis of $P$. parasitica populations with identified SSR markers, a genotype accumulation curve was constructed, which demonstrated that the markers we identified had enough discrimination power to differentiate genotypes in $P$. parasitica populations from Chongqing.

Our results indicated that all of the populations from the two consecutive years were genetically similar to each other. First, the estimation of Weir and Cockerham's coefficient of differentiation accepted the null hypothesis; namely, there was no significant difference among different populations in the same year or between populations of the same place from different years. Second, AMOVA results showed that the major variation was within populations and a low fraction of the variance was attributed to differences among populations. Third, genetic distance analysis showed that all SSR genotypes detected from Chongqing formed a unique cluster being distant from $P$. parasitica strains from Zhejiang Province, China, and Australia. Fourth, PCoA results showed that no clusters were formed, indicating no association between geographic locations and SSR genotype. Finally, the conclusion was also supported by low Nei's genetic distance values $(<0.063)$ between all populations (Table 4$)$.

The occurrence of oospores contributes to the genetic diversity of Phytophthora pathogens (Meng et al. 2014). As to P. parasitica, oospores can be produced by pairing A1 and A2 mating type strains under the laboratory condition (Ko 1978). In the sexually reproducing or recombining populations, the two mating types (A1 and A2) are expected to be in similar frequency (Milgroom 1996). However, in this study, 244 of 245 isolates were of the A2 mating type and no A1 mating type isolates were found. This is consistent with previous reports on the mating type structure of $P$. parasitica populations from tobacco worldwide. In the United States, Parkunan et al. (2010) found that the A2 mating type was dominant (94\%) in Virginia and $6 \%$ belonged to the A1 mating type. Most isolates (56 of 59) were identified as the A2 mating type and three were of A1 mating

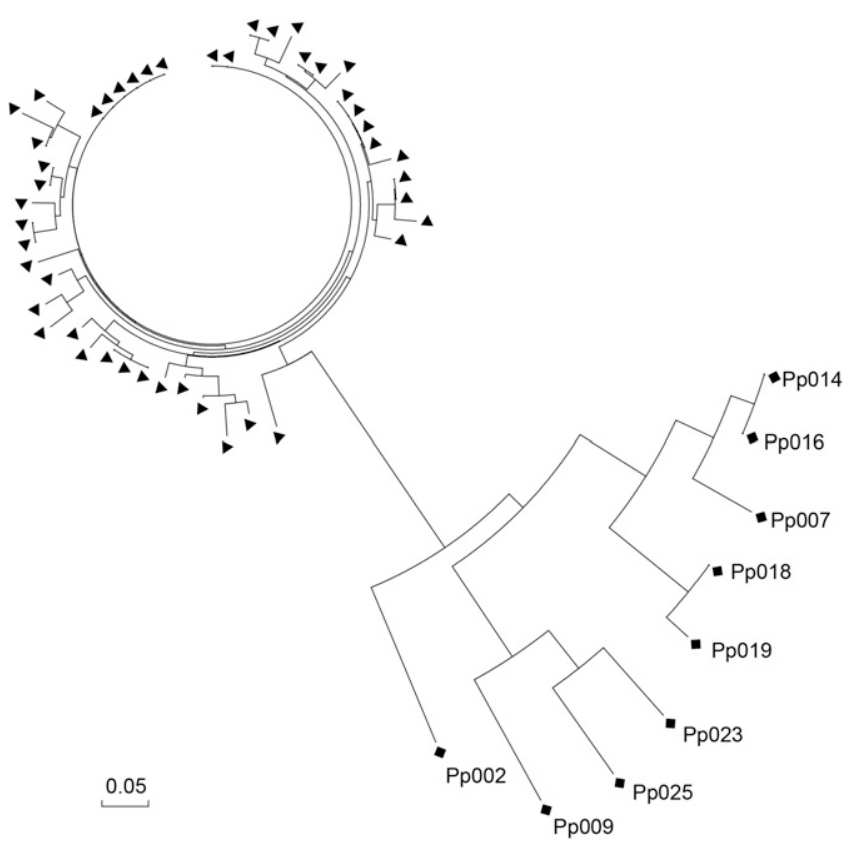

Fig. 4. Genetic relationship analysis of Phytophthora parasitica isolates from Chongqing and that of other regions examined. This analysis was done using the neighbor-joining method based on Bruvo's distance. Filled triangles indicate the 46 multilocus genotypes detected in the Chongqing population and filled squares indicate the nine reference strains (Pp002 to Pp025) from Zhejiang, China, and Australia. type in Georgia (Li et al. 2017). In China, Peng et al. (2011) reported that all $P$. parasitica isolates from Guizhou were of the A2 mating type, which was also predominant in Anhui (Ma and Gao 2006) and Yunnan (Wang et al. 1997). The unequal distribution of mating types suggested that sexual reproduction was unlikely. For all populations analyzed in this research, the observed heterozygosity was higher than the expected heterozygosity, which gave a negative fixation index. In diploid organism, excess heterozygosity often results from extreme clonality (Balloux et al. 2003). Qu (2017) in our laboratory analyzed 34 isolates representing different MLGs from different populations and found that all of the isolates belonged to race 1, indicating that no physiological differentiation was detected in $P$. parasitica populations from Chongqing. Considering all of the above results, $P$. parasitica populations from Chongqing belonged to a clonal lineage and the limited variation might result from random mutation, not from sexual recombination.

Our results showed that $P$. parasitica isolates from Chongqing were metalaxyl sensitive. The $\mathrm{EC}_{50}$ values of $P$. parasitica isolates were all $<1 \mu \mathrm{g} / \mathrm{ml}$, indicating that $P$. parasitica isolates in Chongqing are very sensitive to metalaxyl and this fungicide should control the tobacco black shank. However, excessive use of metalaxyl may result in extensive development of resistant strains, so it is necessary to use this fungicide moderately and use appropriate fungicide resistance management strategies (van den Bosch et al. 2014). We also found a slight variation of $\mathrm{EC}_{50}$ values among isolates examined (from 0.0520 to $0.3320 \mu \mathrm{g} / \mathrm{ml}$ ), which was also found in other regions (Csinos and Bertrand 1994; Morales-Rodríguez et al. 2014; van Jaarsveld et al. 2002); whether there is association between $\mathrm{EC}_{50}$ value variation and geographic locations remains to be investigated in future work.

In summary, 210 SSR loci were identified and 10 were detected to be new polymorphic SSR markers for $P$. parasitica population genetic analysis. $P$. parasitica populations from tobacco fields from Chongqing were determined systematically to be asexual, lacking differentiation among regions, highly sensitive to fungicide metalaxyl and genetically distant from that of other regions examined.

\section{Acknowledgments}

We thank Dr. Kevin Carolan of Rothamsted Research, UK, for fruitful suggestions.

\section{Literature Cited}

Agapow, P. M., and Burt, A. 2001. Indices of multilocus linkage disequilibrium. Mol. Ecol. Notes 1:101-102.

Anderson, J. A., Churchill, G. A., Autrique, J. E., Tanksley, S. D., and Sorrells, M. E. 1993. Optimizing parental selection for genetic linkage maps. Genome 36:181-186.

Balloux, F., Lehmann, L., and de Meeus, T. 2003. The population genetics of clonal and partially clonal diploids. Genetics 164:1635-1644.

Biasi, A., Martin, F., and Schena, L. 2015. Identification and validation of polymorphic microsatellite loci for the analysis of Phytophthora nicotianae populations. J. Microbiol. Methods 110:61-67.

Biasi, A., Martin, F. N., Cacciola, S. O., Lio, G. M. S., Grünwald, N. J., and Schena, L. 2016. Genetic analysis of Phytophthora nicotianae populations from different hosts using microsatellite markers. Phytopathology 106:1006-1014.

Botstein, D., White, R. L., Skolnick, M., and Davis, R. W. 1980. Construction of a genetic linkage map in man using restriction fragment length polymorphisms. Am. J. Hum. Genet. 32:314-331.

Bruvo, R., Michiels, N. K., D’Souza, T. G., and Schulenburg, H. 2004. A simple method for the calculation of microsatellite genotype distances irrespective of ploidy level. Mol. Ecol. 13:2101-2106.

Chen, R. S., and McDonald, B. A. 1996. Sexual reproduction plays a major role in the genetic structure of populations of the fungus Mycosphaerella graminicola. Genetics 142:1119-1127.

Cline, E. T., Farr, D. F., and Rossman, A. Y. 2008. A synopsis of Phytophthora with accurate scientific names, host range, and geographic distribution. Plant Health Prog. 9:32.

Crow, J. F. 1994. Advantages of sexual reproduction. Dev. Genet. 15:205-213.

Csinos, A. S., and Bertrand, P. F. 1994. Distribution of Phytophthora parasitica var. nicotianae races and their sensitivity to metalaxyl in Georgia. Plant Dis. 78:471-474.

da Maia, L. C., Palmieri, D. A., de Souza, V. Q., Kopp, M. M., de Carvalho, F. I. F., and Costa de Oliveira, A. C. 2008. SSR Locator: Tool for simple sequence repeat discovery integrated with primer design and PCR simulation. Int. J. Plant Genomics 2008:412696. 
Eyre, C. A., Kozanitas, M., and Garbelotto, M. 2013. Population dynamics of aerial and terrestrial populations of Phytophthora ramorum in a California forest under different climatic conditions. Phytopathology 103:1141-1152.

Flier, W. G., Grünwald, N. J., Kroon, L. P., Sturbaum, A. K., van den Bosch, T. B., Garay-Serrano, E., Lozoya-Saldaña, H., Fry, W. E., and Turkensteen, L. J. 2003. The population structure of Phytophthora infestans from the Toluca Valley of central Mexico suggests genetic differentiation between populations from cultivated potato and wild Solanum spp. Phytopathology 93: 382-390.

Gallup, C., Sullivan, M., and Shew, H. 2006. Black shank of tobacco. Plant Health Instructor.

Goodwin, S. B., Drenth, A., and Fry, W. E. 1992. Cloning and genetic analyses of two highly polymorphic, moderately repetitive nuclear DNAs from Phytophthora infestans. Curr. Genet. 22:107-115.

Grünwald, N. J., Flier, W. G., Sturbaum, A. K., Garay-Serrano, E., van den Bosch, T. B., Smart, C. D., Matuszak, J. M., Lozoya-Saldaña, H., Turkensteen, L. J., and Fry, W. E. 2001. Population structure of Phytophthora infestans in the Toluca Valley region of central Mexico. Phytopathology 91:882-890.

Grünwald, N. J., Goodwin, S. B., Milgroom, M. G., and Fry, W. E. 2003. Analysis of genotypic diversity data for populations of microorganisms. Phytopathology 93:738-746

Ivors, K., Garbelotto, M., Vries, I. D. E., Ruyter-Spira, C., Hekkert, B. T. E., Rosenzweig, N., and Bonants, P. 2006. Microsatellite markers identify three lineages of Phytophthora ramorum in US nurseries, yet single lineages in US forest and European nursery populations. Mol. Ecol. 15: 1493-1505.

Kamoun, S., Furzer, O., Jones, J. D. G., Judelson, H. S., Ali, G. S., Dalio, R. J. D., Roy, S. G., Schena, L., Zambounis, A., Panabières, F., Cahill, D., Ruocco, M., Figueiredo, A., Chen, X.-R., Hulvey, J., Stam, R., Lamour, K., Gijzen, M., Tyler, B. M., Grünwald, N. J., Mukhtar, M. S., Tomé, D. F. A., Tör, M., Ackerveken, G. V. D., Mcdowell, J., Daayf, F., Fry, W. E., LindqvistKreuze, H., Meijer, H. J. G., Petre, B., Ristaino, J., Yoshida, K., Birch, P. R. J., and Govers, F. 2015. The top 10 oomycete pathogens in molecular plant pathology. Mol. Plant Pathol. 16:413-434.

Kamvar, Z. N., Tabima, J. F., and Grünwald, N. J. 2014. Poppr: An R package for genetic analysis of populations with clonal, partially clonal, and/or sexual reproduction. PeerJ 2:e281.

Kang, Y. G. 2000. In vitro sensitivity to metalaxyl of Phytophthora parasitica var. nicotianae isolates from burley tobacco in Korea. Plant Pathol. J. 16:222-226.

Ko, W. H. 1978. Heterothallic Phytophthora: Evidence for hormonal regulation of sexual reproduction. Microbiology 107:15-18

Li, Y., Cooke, D. E., Jacobsen, E., and van der Lee, T. 2013. Efficient multiplex simple sequence repeat genotyping of the oomycete plant pathogen Phytophthora infestans. J. Microbiol. Methods 92:316-322.

Li, Y., Harris-Shultz, K., Wang, H., Wadl, P. A., and Ji, P. 2017. Population structure and genetic diversity of Phytophthora nicotianae from tobacco in Georgia. Plant Dis. 101:1113-1118.

Li, Y., van der Lee, T. A., Evenhuis, A., van den Bosch, G. B., van Bekkum, P. J., Forch, M. G., van Gent-Pelzer, M. P., van Raaij, H. M., Jacobsen, E., Huang, S. W., Govers, F., Vleeshouwers, V. G., and Kessel, G. J. 2012. Population dynamics of Phytophthora infestans in the Netherlands reveals expansion and spread of dominant clonal lineages and virulence in sexual offspring. G3 (Bethesda) 2:1529-1540.

Ma, G., and Gao, Z. 2006. Mating types and distribution of Phytophthora nicotianae var. nicotianae in Anhui. Acta Phytopathol. Sin. 36:566-568 (in Chinese with English abstract).

Meng, Y., Zhang, Q., Ding, W., and Shan, W. 2014. Phytophthora parasitica: A model oomycete plant pathogen. Mycology 5:43-51.

Milgroom, M. G. 1996. Recombination and the multilocus structure of fungal populations. Annu. Rev. Phytopathol. 34:457-477.

Montarry, J., Andrivon, D., Glais, I., Corbiere, R., Mialdea, G., and Delmotte, F. 2010. Microsatellite markers reveal two admixed genetic groups and an ongoing displacement within the French population of the invasive plant pathogen Phytophthora infestans. Mol. Ecol. 19:1965-1977.

Montes, M., Nielsen, B. J., Schmidt, S., Bødker, L., Kjøller, R., and Rosendahl, S. 2016. Population genetics of Phytophthora infestans in Denmark reveals dominantly clonal populations and specific alleles linked to metalaxyl-M resistance. Plant Pathol. 65:744-753.
Morales-Rodríguez, C., Palo, C., Palo, E., and Rodríguez-Molina, M. C. 2014 Control of Phytophthora nicotianae with mefenoxam, fresh Brassica tissues, and Brassica pellets. Plant Dis. 98:77-83.

Nei, M. 1972. Genetic distance between populations. Am. Nat. 106:283-292.

Nei, M. 1973. Analysis of gene diversity in subdivided populations. Proc. Natl Acad. Sci. U.S.A. 70:3321-3323.

Onions, A. H. 1971. Preservation of Fungi. Pages 113-151 in: Methods in Microbiology, vol. 4. C. Booth, ed. Academic Press, New York.

Pagliaccia, D., Pond, E., McKee, B., and Douhan, G. W. 2013. Population genetic structure of Phytophthora cinnamomi associated with avocado in California and the discovery of a potentially recent introduction of a new clonal lineage. Phytopathology 103:91-97.

Parker, P. G., Snow, A. A., Schug, M. D., Booton, G. C., and Fuerst, P. A. 1998 What molecules can tell us about populations: Choosing and using a molecular marker. Ecology 79:361-382

Parkunan, V., Johnson, C. S., Bowman, B. C., and Hong, C. X. 2010. Population structure, mating type, and mefenoxam sensitivity of Phytophthora nicotianae in Virginia tobacco fields. Plant Dis. 94:1361-1365.

Peakall, R., and Smouse, P. E. 2006. GENALEX 6: Genetic analysis in Excel Population genetic software for teaching and research. Mol. Ecol. Notes 6:288-295.

Peakall, R., and Smouse, P. E. 2012. GenAlEx 6.5: Genetic analysis in Excel. Population genetic software for teaching and research - an update. Bioinformatics 28:2537-2539.

Peng, L., Ding, H., and Ge, Y. 2011. Mating type and distribution of Phytophthora nicotianae in Guizhou province. J. Henan Agric. Sci. 11:93-96 (in Chinese with English abstract).

Qian, N. 2011. The genetic diversity of Phytophthora nicotiana in Chongqing. Master's dissertation, Southwest University, Chongqing, China (in Chinese with English abstract).

Qu, Y. 2017. Physiological differentiation and resistance to main fungicides of tobacco black shank pathogen in Chongqing. Master's dissertation, Northwest A\&F University, Yangling, China (in Chinese with English abstract).

Simpson, E. H. 1949. Measurement of diversity. Nature 163:688.

Sun, C. 2009. Mating type and physiological race of Phytophthora nicotiana in Chongqing. Master's dissertation, Southwest University, Chongqing, China (in Chinese with English abstract)

Tamura, K., Stecher, G., Peterson, D., Filipski, A., and Kumar, S. 2013. MEGA6: Molecular evolutionary genetics analysis version 6.0. Mol. Biol. Evol. 30: 2725-2729.

Tian, Y., Sun, J., Li, H., Wang, G., Ma, Y., Liu, D., Quan, J., and Shan, W. 2015a Dominance of a single clonal lineage in the Phytophthora infestans population from northern Shaanxi, China revealed by genetic and phenotypic diversity analysis. Plant Pathol. 64:200-206.

Tian, Y., Yin, J., Sun, J., Ma, H., Ma, Y., Quan, J., and Shan, W. 2015b. Population structure of the late blight pathogen Phytophthora infestans in a potato germplasm nursery in two consecutive years. Phytopathology 105:771-777.

Tian, Y. E., Yin, J. L., Sun, J. P., Ma, Y. F., Wang, Q. H., Quan, J. L., and Shan, W. X. 2016. Population genetic analysis of Phytophthora infestans in northwestern China. Plant Pathol. 65:17-25.

Untergasser, A., Cutcutache, I., Koressaar, T., Ye, J., Faircloth, B. C., Remm, M., and Rozen, S. G. 2012. Primer3-new capabilities and interfaces. Nucleic Acids Res. 40:e115.

van den Bosch, F., Oliver, R., van den Berg, F., and Paveley, N. 2014. Governing principles can guide fungicide-resistance management tactics. Annu. Rev. Phytopathol. 52:175-195.

van Jaarsveld, E., Wingfield, M., and Drenth, A. 2002. Effect of metalaxyl resistance and cultivar resistance on control of Phytophthora nicotianae in tobacco. Plant Dis. 86:362-366.

Wang, G., Zheng, X., Lu, J., and Li, T. 1997. Mating type and distribution of the caused organism of the tobacco black shank in Yunnan province. J. Nanjing Agric. Univ. 20:31-34 (in Chinese with English abstract).

Wang, H. C., Chen, X. J., Cai, L. T., Cao, Y., Lu, N., Xia, H. Q., Wang, M. S., and Shang, S. H. 2013. Race distribution and distribution of sensitivities to mefenoxam among isolates of Phytophthora parasitica var. nicotianae in Guizhou province of China. Crop Prot. 52:136-140.

Yeh, F. C., Yang, R. C., Boyle, T. B., Ye, Z., and Mao, J. X. 1997. POPGENE, the user-friendly shareware for population genetic analysis. Molecular Biology and Biotechnology Centre, University of Alberta, Canada. 